\title{
Polarization-Resolved Modulation Response of Single-Transverse-Mode Vertical-Cavity Surface-Emitting Lasers
}

\author{
M. S. Torre and C. Masoller
}

\begin{abstract}
The small-signal modulation response of vertical-cavity surface-emitting lasers (VCSELs) is studied numerically, based on the spin-flip model. A detailed characterization of the influence of various parameters, such as the dichroism, the birefringence, the spin-flip rate and the noise level is done. The analysis is performed in regions of the parameter space where the operating conditions, in the absence of modulation, are such that there is either stable single linear polarization, or bistability of two orthogonal linear polarizations or polarization instability. In the instability region the intensities of the orthogonal polarizations display anticorrelated self-sustained oscillations that result in a complex small-signal modulation response, exhibiting multiple resonance peaks with frequencies that vary with the injection current and the spin-flip rate. In contrast, in parameter regions where there is stable single polarization the modulation response of the total intensity is as that of a single-mode laser, exhibiting a conventional single-resonance peak, and is almost not influenced by the value of the spin-flip rate. Polarization mode-hopping results in a distortion of the polarization-resolved modulation response at low frequencies.
\end{abstract}

Index Terms-Laser diodes, modulation response, polarization switching, semiconductor lasers, vertical-cavity surface-emitting lasers (VCSELs).

\section{INTRODUCTION}

$\mathbf{V}$ ERTICAL-CAVITY surface-emitting lasers (VCSELs) have several characteristics that make them very attractive light sources for the next generation of Ethernet, local area networks, and optical interconnects [1], [2]. They have low threshold currents and circular output beams that result in high coupling efficiencies into optical fibers. They offer single-longitudinal-mode emission over a wide mode-hoping-free tuning range. VCSELs emitting in the long-wavelength-region (1.3 and $1.55 \mu \mathrm{m}$ ) have reached a degree of performance which allows to enter industrial applications, providing cost efficiency at wavelengths of interest for mature data links and optical fiber technologies. Room-temperature high-speed and error-free

Manuscript received May 13, 2008; revised June 18, 2008. Current version published January 21, 2009. The work of M. S. Torre was supported in part by CONICET Grant PIP 6474, FONCyT Grant 3/09598. The work of C. Massoler was supported in part by the "Ramon y Cajal" Program (Spain) and the Spanish Ministerio de Educacion y Ciencia through project FIS2005-07931C03-03.This work was supported in part by the U.S. Air Force Office of Scientific Research under Grant FA9550-07-1-0238.

M. S. Torre is with the Instituto de Física “Arroyo Seco," U.N.C.P.B.A, Pinto 399 (7000) Tandil, Argentina.

C. Masoller is with the Departament de Fisica i Enginyeria Nuclear, Universitat Politecnica de Catalunya, E-08222 Terrassa, Barcelona, Spain.

Digital Object Identifier 10.1109/JQE.2008.2002669 data transmission has been demonstrated [3], [4]. Monolithically integrated one and two dimensional arrays, suitable for multichannel optical interconnects are being fabricated using lithographic techniques [5].

The modulation characteristics of free-running and injection-locked VCSELs are nowadays being intensively investigated [6]. A $40 \mathrm{GHz}$ bandwidth and $64 \mathrm{GHz}$ resonance frequency was recently reported, for a directly modulated, injection-locked $1.55 \mu \mathrm{m}$ InGaAlAs-InP buried tunnel junction VCSEL [7]. Methods for achieving a wide and tailorable modulation bandwidth are continuously being developed. To potentially achieve ultrahigh modulation bandwidth $(>100 \mathrm{GHz})$ a novel configuration of two cascaded injection-locked VCSELs was recently proposed [8], and a $66 \mathrm{GHz}$ bandwidth was demonstrated using VCSELs with an original, free-running 10 $\mathrm{GHz}$ bandwidth.

Optically injection-locked lasers, both edge-emitting lasers and VCSELs have, however, the disadvantage of a significant reduction in the modulation efficiency in between low frequencies and the resonance frequency. Here we propose theoretically a possible way to engineering the modulation response of $\mathrm{VC}$ SELs, in the low frequency range, by exploiting the intrinsic nonlinearities in the interaction of two orthogonal linear polarization modes, that are associated with the same longitudinal mode and the same fundamental transverse mode of the VCSEL.

A free-running VCSEL is numerically investigated using the well-known spin-flip model [9]-[11]. The spin-flip model considers the slowly-varying complex amplitudes of two orthogonal circularly polarized components of the optical field, $E^{+}$and $E^{-}$, coupled to two carrier densities with opposite spin value, $\mathrm{N}^{+}$and $\mathrm{N}^{-}$. Material and/or cavity anisotropies (dichroism and birefringence) result in solutions of the model corresponding to two orthogonal linearly polarized states with different emission frequencies, referred to as $x$ and $y$. In the parameter space there are domains of bistability, monostability and polarization instability. Elliptically polarized states are also possible (both orthogonal linearly polarized states having the same emission frequency). Our simulations show that inside the domain of polarization dynamical instability the modulation response exhibits multiple resonance peaks, whose frequencies vary with the injection current and the spin-flip rate (the spin-flip rate, $\gamma_{s}$, models the equilibration of the populations with opposite spin, due to a variety of microscopic processes termed spin-flip relaxation processes, and is temperature dependent). These extra resonances occur for values of the spin-flip rate that are not too large (typically $\gamma_{s}<20 \mathrm{~ns}^{-1}$ ) and arise 
from anticorrelated self-sustained oscillations of the two linear polarizations.

To the best of our knowledge, this is the first report of the modulation response of free-running VCSELs exhibiting a rich structure due to polarization competition; previously reported multiresonance peaks arise due to multitransverse mode competition [12]-[15].

We also demonstrate that within regions of stable single polarization the modulation response is as that of a single-mode laser, and is rather unaffected by the value of the spin-flip rate. In particular, we assess the influence of polarization mode hopping, that occurs in domains of bistability, if spontaneous emission noise is large enough. We find that polarization hopping results in a degradation of the flat response at low frequencies. This distortion is more pronounced in the polarization-resolved modulation responses, as compared with the distortion in the response of the total intensity.

In a previous work [16] we investigated the small and largesignal response of current-modulated VCSELs employing the spin-flip model, extended to account for transverse nonuniform optical modes and the effects of the diffusion of carriers. It is well-known that transverse inhomogeneities have an important impact on the small-signal response [14], [17]. A main conclusion in our previous work [16] was that carrier diffusion and transverse inhomogeneities affect the response of the total intensity, but have little impact on the underlying polarization competition, which is mainly determined by the combined effects of cavity anisotropies (dichroism and birefringence), saturable dispersion (alpha-factor), the spin-flip relaxation rate, and the noise strength. Thus, here we consider the spin-flip model without accounting for transverse effects. This permits faster numerical simulations of the model equations, that are cost efficient for studying the influence of the various parameters that determine the polarization state of the laser output.

This paper is organized as follows. The model is well-known and is presented briefly in Section II. Section III presents the numerically calculated modulation response in different parameter regions, corresponding to monostability, bistability and instability. The influence of various model parameters (the dichroism, the birefringence, the spin-flip rate and the noise strength) is discussed. Section IV contains a summary and the conclusions.

\section{THE MODEL}

The rate equations for the linearly polarized slowly-varying complex amplitudes, $E_{x}$ and $E_{y}$, the total carrier density, $N=$ $N_{+}+N_{-}$, and the carrier difference, $n=N_{+}-N_{-}$(where $N_{+}$ and $N_{-}$are two carrier populations with positive and negative spin value) are [9]-[11]

$$
\begin{aligned}
\frac{d E_{x}}{d t}= & k(1+i \alpha)\left[(N-1) E_{x}+i n E_{y}\right] \\
& -\left(\gamma_{a}+i \gamma_{p}\right) E_{x}+\sqrt{\beta_{\mathrm{sp}}} \xi_{x} \\
\frac{d E_{y}}{d t}= & k(1+i \alpha)\left[(N-1) E_{y}-i n E_{x}\right] \\
& +\left(\gamma_{a}+i \gamma_{p}\right) E_{y}+\sqrt{\beta_{\mathrm{sp}}} \xi_{y},
\end{aligned}
$$

$$
\begin{aligned}
\frac{d N}{d t}= & \gamma_{N}\left[\mu(t)-N\left(1+\left|E_{x}\right|^{2}+\left|E_{y}\right|^{2}\right)\right. \\
& \left.-i n\left(E_{y} E_{x}^{*}-E_{x} E_{y}^{*}\right)\right], \\
\frac{d n}{d t}= & -\gamma_{s} n-\gamma_{N}\left[n\left(\left|E_{x}\right|^{2}+\left|E_{y}\right|^{2}\right)\right. \\
& \left.+i N\left(E_{y} E_{x}^{*}-E_{x} E_{y}^{*}\right)\right] .
\end{aligned}
$$

Here $k$ is the field decay rate, $\gamma_{N}$ is the decay rate of the total carrier population, $\gamma_{s}$ is the spin-flip rate (which accounts for the mixing of carrier populations with different spins), $\alpha$ the linewidth enhancement factor, $\gamma_{a}$ and $\gamma_{p}$ are linear anisotrophies (representing dichroism and birefringence), and $\mu$ is the normalized injection current parameter (the static threshold is $\left.\mu_{\mathrm{th}}=1\right)$. The current parameter is modulated in time as

$$
\mu(t)=\mu_{\mathrm{dc}}+A_{m} \cos \left(2 \pi f_{m} t\right),
$$

where $\mu_{\mathrm{dc}}$ is the dc-value, $A_{m}$ is the modulation depth and $f_{m}$ is the modulation frequency.

In the absence of current modulation, the steady-state solutions of the model corresponding to linearly polarized states are

$$
E_{x}=\mathcal{E}_{x} e^{i \omega_{x} t}, \quad E_{y}=0, \quad N=\mathcal{N}_{x}, \quad n=0,
$$

and

$$
E_{x}=0, E_{y}=\mathcal{E}_{y} e^{i \omega_{y} t}, \quad N=\mathcal{N}_{y}, \quad n=0
$$

where $\omega_{x, y}= \pm \alpha \gamma_{a} \mp \gamma_{p}, \mathcal{N}_{x, y}=1 \pm \gamma_{a} / k$, and $\mathcal{E}_{x, y}=$ $\sqrt{\mu / \mathcal{N}_{x, y}-1}$. Solutions corresponding to elliptically polarized states also exist [10]. The linear stability analysis reveals that the parameter space divides into regions of mono-stability (only one linear polarization is stable), regions of bistability (both linear polarizations are stable), and regions of polarization instability (both linear polarizations are unstable). In the following section we simulate the model equations considering a small amplitude current modulation and analyze the modulation response in these different regions.

\section{NUMERICAL RESULTS}

We simulated the model equations with typical VCSEL parameters [10], [19]-[21]: $k=300 \mathrm{~ns}^{-1}, \alpha=3, \gamma_{N}=1$ $\mathrm{ns}^{-1}$. The noise level, unless otherwise stated, is $\beta_{\mathrm{sp}}=10^{-6}$ $\mathrm{ns}^{-1}$. We use two sets of parameters for the linear anisotrophies: $\gamma_{a}= \pm 0.2 \mathrm{~ns}^{-1}, \gamma_{p}=3$ and $100 \mathrm{rad} / \mathrm{ns} . \gamma_{a}>0$ gives the $y$ polarization, that is the high-frequency polarization, a lower threshold; $\gamma_{a}<0$ gives the low-frequency $x$ polarization a lower threshold. The two values of $\gamma_{p}$ represent small and large birefringent VCSELs (the frequency split between the two linear polarizations being about 1 and $30 \mathrm{GHz}$ respectively). $\gamma_{a}$ and $\gamma_{p}$ are combined such as to consider parameters where there is nontrivial polarization dynamics.

1) For negative dichroism the low frequency $x$ polarization turns-on at threshold and, if $\gamma_{p}$ is small enough, a polarization switching (PS) to the high frequency $y$ polarization occurs for increasing injection current. This PS has been referred to as type II [18].

2) For positive dichroism the high frequency $y$ polarization turns-on at threshold and, if $\gamma_{p}$ is large enough, a PS to the low frequency $x$ polarization occurs for increasing injection current (type I PS [18]). 

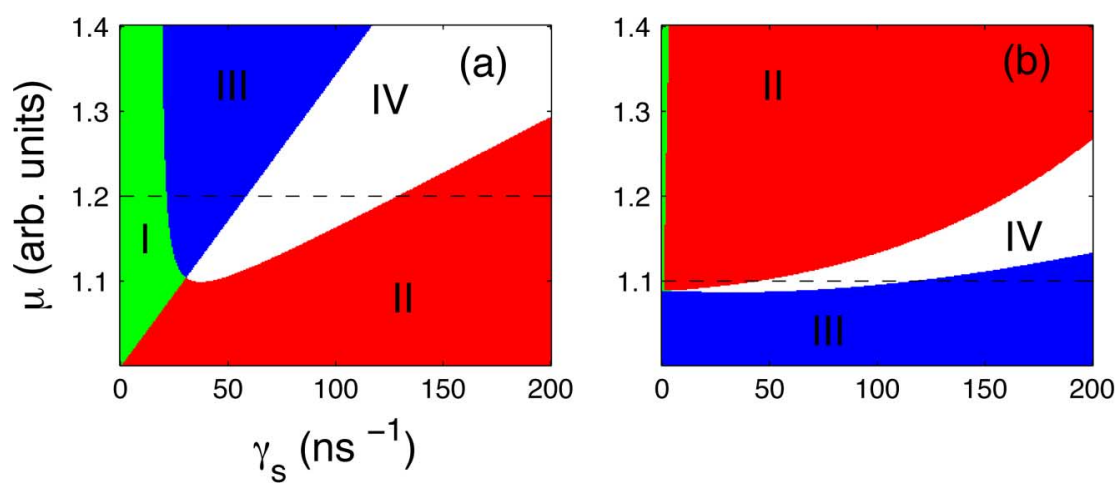

Fig. 1. Stability regions of the linearly polarized solutions. (a) $\gamma_{a}=-0.2 \mathrm{~ns}^{-1}, \gamma_{p}=3 \mathrm{rad} / \mathrm{ns}$. (b) $\gamma_{a}=0.2 \mathrm{~ns}^{-1}, \gamma_{p}=100 \mathrm{rad} / \mathrm{ns}$. The dashed lines indicate the regions studied numerically.

Fig. 1(a) and (b) displays the linear stability of the $x$ and $y$ polarizations in the parameter space $\left(\gamma_{s}, \mu\right)$, for anisotropy parameters corresponding to type II PS and type I PS respectively. The stability of the $x$ and $y$ polarizations was calculated by solving the characteristic equation for the eigenvalues, (44) in [10]. The lines separating the stability regions in the parameter space are those for which the real part of an eigenvalue is equal to zero.

It can be observed that the parameter space is divided into four different regions. In I both linear polarizations are unstable; in II and III there is monostability: only $x$ or $y$ is stable; in IV there is bistability (both linear polarizations are stable). Near the threshold there is monostability: $x$ is stable in Fig. 1(a) while $y$ is stable in Fig. 1(b). The range of injection current values for monostable polarization increases with $\gamma_{s}$ in Fig. 1(a) while is rather independent of $\gamma_{s}$ in Fig. 1(b).

In the following we investigate the influence of small-amplitude current modulation in these different regions. We focus on assessing the role of the spin-flip rate, and thus vary $\gamma_{s}$ while keeping other parameters constant.

We calculate the small-signal modulation response integrating numerically the full model equations, (1)-(4), without linearization. The time-traces of the intensities of the two linear polarizations, $I_{x, y}(t)=\left|E_{x, y}(t)\right|^{2}$, and of the total intensity, $I_{T}(t)=I_{x}(t)+I_{y}(t)$, were Fourier transformed and we selected the spectral component at the modulation frequency, $\tilde{I}_{T, x, y}\left(\nu=f_{m}\right)$. The modulation response was normalized to the response of the total intensity at the lowest frequency, i.e., we computed $\tilde{I}_{T, x, y}\left(f_{m}\right) / \tilde{I}_{T}(0.01 \mathrm{GHz})$.

\section{A. Negative Dichroism and Small Birefringence}

We consider anisotropy parameters $\gamma_{a}$ and $\gamma_{p}$ in the parameter region of type II PS (the $x$ polarization is stable close to threshold). We increase the value of the spin-flip rate, keeping all other parameters constant. This corresponds to an horizontal scan in Fig. 1(a). We chose $\mu_{\mathrm{dc}}=1.2$ in order to find different regions along the scan.

First, we describe the dynamics in the absence of current modulation. The time evolution of the total intensity and of the $x$ and $y$ polarizations is displayed in Fig. 2. For low $\gamma_{s}$ no linear polarization is stable and we observe oscillations of the intensities of the two polarizations [Fig. 2(a) and (b)]. The oscillations are anticorrelated but are not perfectly in antiphase, and thus the total intensity also displays self-sustained oscillations [as shown

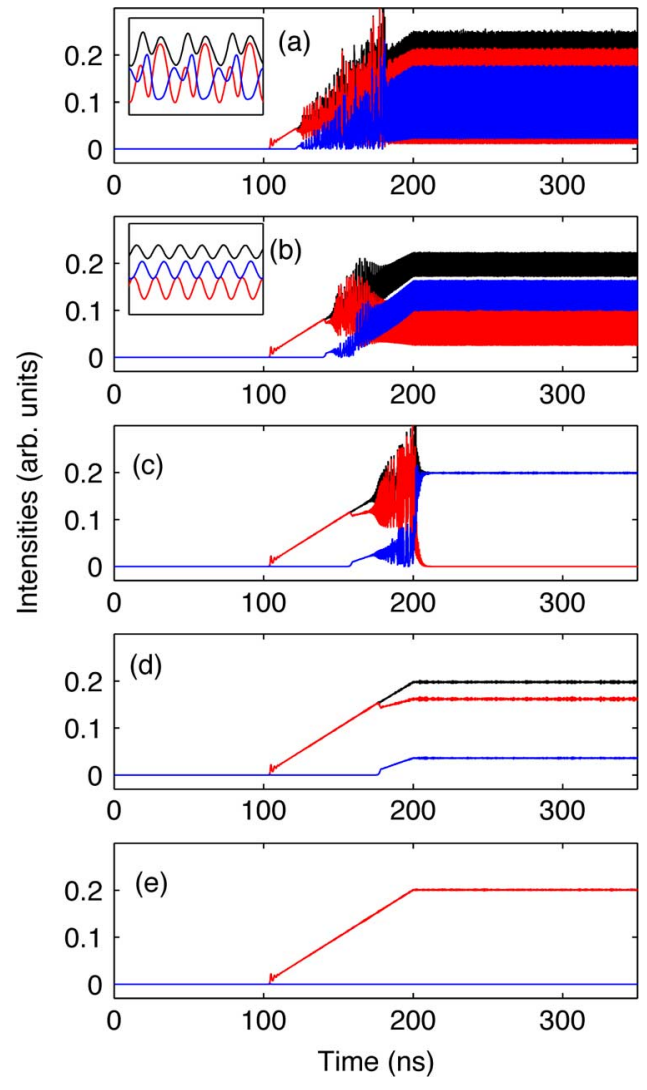

Fig. 2. Time traces of the total intensity, and of the $x$ and $y$ polarizations in the absence of current modulation, for various values of the spin-flip rate: $10 \mathrm{~ns}^{-1}$ (a); $20 \mathrm{~ns}^{-1}$ (b); $30 \mathrm{~ns}^{-1}$ (c); $40 \mathrm{~ns}^{-1}$ (d); $120 \mathrm{~ns}^{-1}$ (e). Other parameters $\gamma_{a}=-0.2 \mathrm{~ns}^{-1}, \gamma_{p}=3 \mathrm{rad} / \mathrm{ns}, \mu=1.2, \beta_{\mathrm{sp}}=10^{-6} \mathrm{~ns}^{-1}$. The total intensity is plotted with a black line, the $x$ polarization with a red line, and the $y$ polarization with a blue line.

in detail in the insets of Fig. 2(a) and 2(b)]. As $\gamma_{s}$ increases the following regimes are predicted from the linear stability analysis: the $y$ polarization is stable [Fig. 2(c)], for larger $\gamma_{s}$ both polarizations are stable, and the output is either $x$ or $y$ polarized depending on the transient laser turn-on. Near the borders of the bistability region, in addition to the linearly polarized states, elliptically polarized states are also stable [Fig. 2(d)]. For even larger $\gamma_{s}$, only the $x$ polarization is stable [Fig. 2(e)]. The representation of these states using the Poincare Sphere results in fixed points and limit cycles, as shown in Fig. 5 of [10], and in 


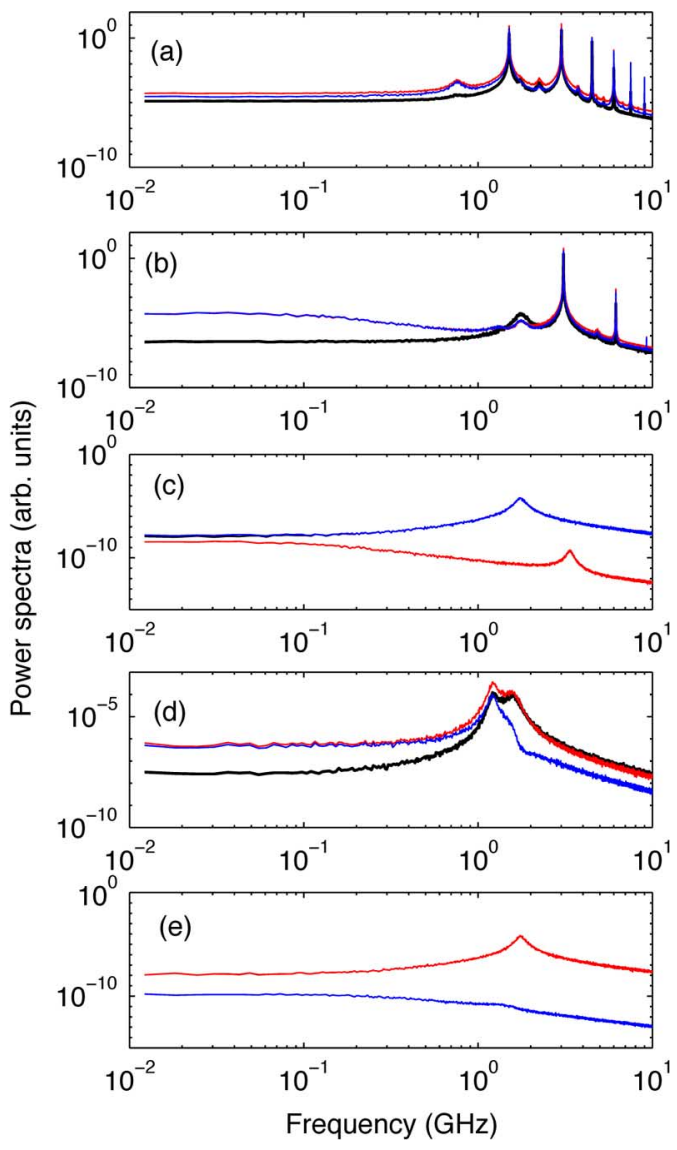

Fig. 3. Polarization-resolved power spectrum (total intensity: thick line, $x$ and $y$ intensities: thin lines, color online: $x$ red and $y$ blue). Parameters are as in Fig. 2.

Figs. 2 and 3 of [19], that were done for similar parameters as Fig. 2.

The corresponding power spectra of the total and the modal intensities are displayed in Fig. 3, and the modulation responses, in Fig. 4. For low $\gamma_{s}$, Fig. 3(a) and (b), several peaks are observed in the spectra of the linear polarizations and also in the spectrum of the total intensity. These peaks result in complex modulation responses, shown in Fig. 4(a) and (b). The characteristic single-peaked response is observed only when the output is linearly polarized [Fig. 4(c) and (e)]. In contrast, when there is dynamic instability or elliptic polarization there is a multipeaked modulation response of the total intensity and of both orthogonal polarizations. In the region of bistability the modulation response is single-peaked but there is a distortion at low frequencies, as is discussed in the next subsection.

We note that the modulation-response and the relative intensity noise (RIN) are related in the limit of small signals, and thus is interesting to compare the results presented here with the RIN calculated analytically and numerically in [19]: the RINs displayed in Fig. 3(b) and (e) of [19] compare well with the modulation response displayed in Fig. 4(c) and (e) (dominant polarization: $y$ and $x$ respectively), while the multipeaked response displayed in Fig. 4(a), (b), and (d) occurs for parameters that were not analyzed in [19].

We investigated the variation of the frequency of the main resonance with the injection current dc value and with the
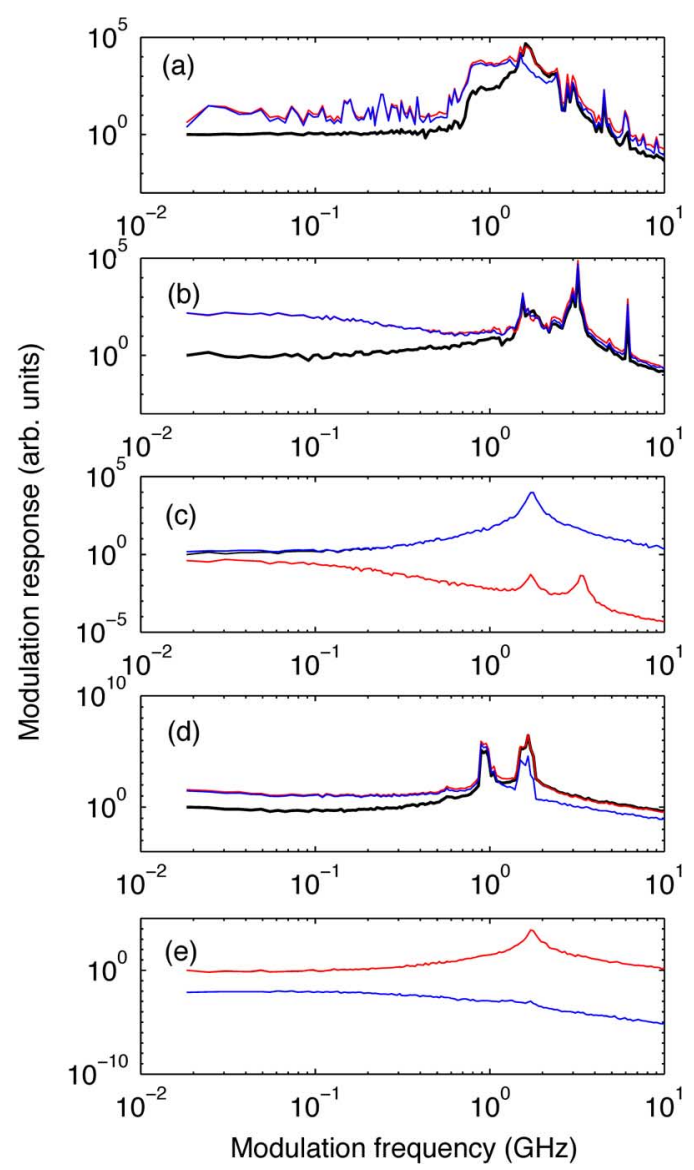

Fig. 4. Modulation response (total intensity: thick line, $x$ and $y$ intensities: thin lines, color online: $x$ red and $y$ blue). Parameters are as in Fig. 2, and $A_{m}=$ 0.01 .

spin-flip rate. Fig. 5(a) displays the frequency of the main peak in the spectrum of the total intensity, as a function of $\mu_{\mathrm{dc}}$, for two values of the spin flip rate. The frequency increases with the injection current, but non-monotonically, i.e., there are some abrupt variations that often are accompanied by changes in the dominant polarization, as shown in Fig. 5(b), where it can also be observed that the frequency of the peak varies with the square-root of $\mu_{\mathrm{dc}}-1$, in the same way as the relaxation oscillation frequency. We note that our results are in agrement with the observations of Sondermann et al. [20] where a kink in the plot of the relaxation oscillation frequency versus current was observed at the PS point (see [20, Fig. 2]).

In regions where the emission is linearly polarized, the modulation response is almost independent of the value of the spin-flip rate. This is shown in Fig. 6, that compares the modulation response when the emission is $y$ polarized (for $\gamma_{s}=30 \mathrm{~ns}^{-1}$ ) and when is $x$ polarized (for $\gamma_{s}=120 \mathrm{~ns}^{-1}$ ).

\section{B. Positive Dichroism and Large Birefringence}

Let us now consider anisotropy parameters in the parameter region of type I PS (the $y$ polarization is stable at threshold). Again, we increase the value of the spin-flip rate, keeping other parameters constant, and this corresponds to an horizontal scan in Fig. 1(b). We chose $\mu_{\mathrm{dc}}=1.1$ in order to find different regions along the scan. 

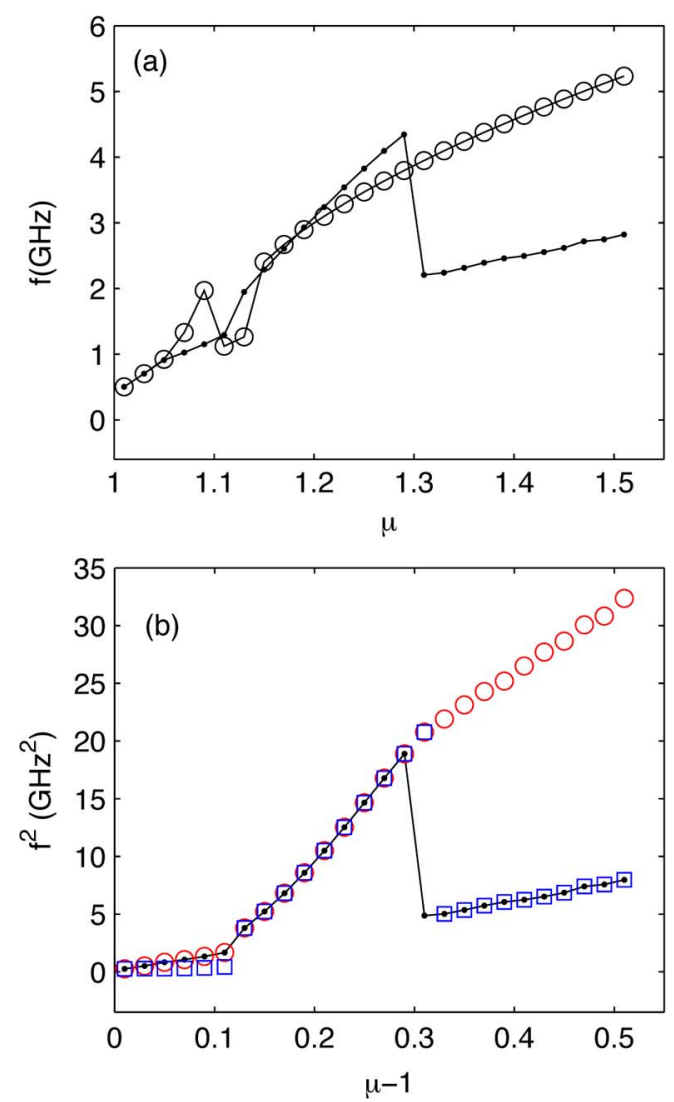

Fig. 5. (a) Frequency of the main peak in the power spectrum of the total intensity for $\gamma_{s}=10 \mathrm{~ns}^{-1}$ (circles) and $20 \mathrm{~ns}^{-1}$ (dots). (b) Frequency of the main peak in the power spectrum of the $x$ polarization (circles), the $y$ polarization (squares) and the total intensity (dots) for $\gamma_{s}=20 \mathrm{~ns}^{-1}$.

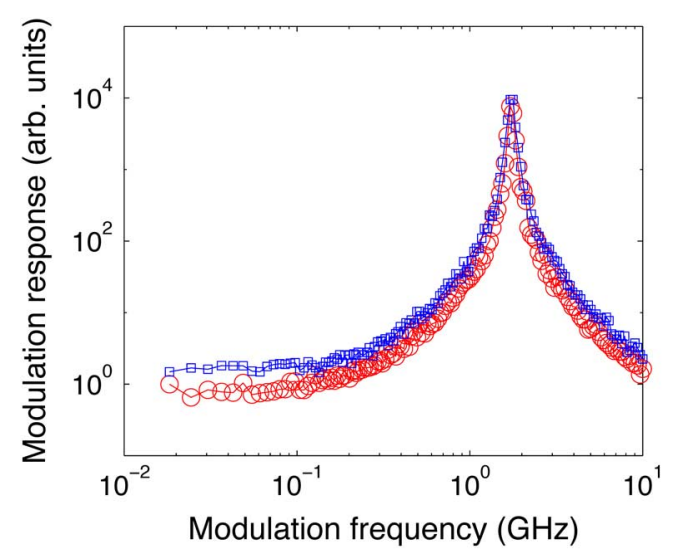

Fig. 6. Modulation response in parameter regions of polarization monostability: for $\gamma_{s}=30 \mathrm{~ns}^{-1}$ (squares) the $y$ polarization turns on; for $\gamma_{s}=120$ $\mathrm{ns}^{-1}$ the $x$ polarization turns on (circles).

The following regimes are predicted from the linear stability analysis: for very low $\gamma_{s}$ there is polarization instability; however, these values are too low to be considered realistic for practical VCSELs. We start the scan at values of $\gamma_{s}$ where the $x$ polarization is stable. For larger $\gamma_{s}$ both polarizations are stable, and if the noise is not too strong, there is stable emission in either $x$ or $y$ polarization (with strong noise levels there is polarization mode hopping as discussed below). For even larger $\gamma_{s}$ only

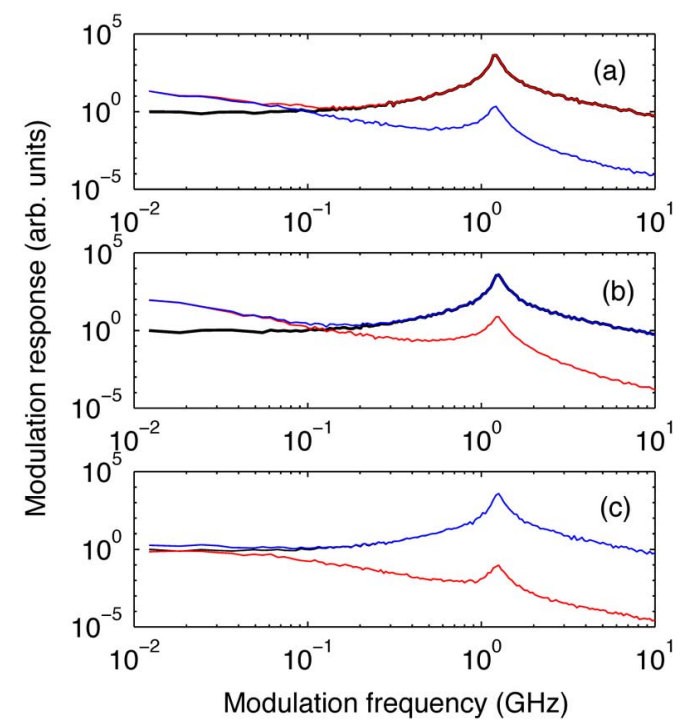

Fig. 7. Modulation response for positive dichroism and large birefringence. $\gamma_{s}=10 \mathrm{~ns}^{-1}$ (a); $100 \mathrm{~ns}^{-1}(\mathrm{~b}) ; 200 \mathrm{~ns}^{-1}$ (c). The noise strength is $\beta_{\mathrm{sp}}=$ $10^{-6} \mathrm{~ns}^{-1}$, other parameters are indicated in the text.

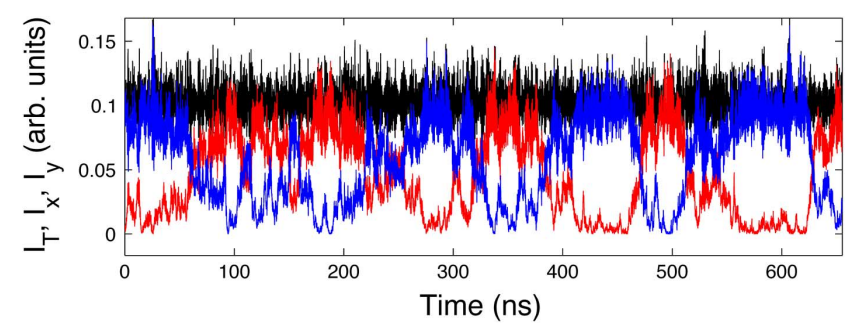

Fig. 8. Time traces of the total and modal intensities in the stochastic mode hopping regime. Parameter are as in Fig. 7 (b) $\left(\gamma_{s}=100 \mathrm{~ns}^{-1}\right)$ and the noise level is $\beta_{\mathrm{sp}}=10^{-3} \mathrm{~ns}^{-1}$.

the $y$ polarization is stable. The polarization-resolved modulation response, displayed in Fig. 7, is single-peaked in the three regions and the resonance occurs at the same frequency (that is the relaxation oscillation frequency) for both linear polarizations. Comparing Fig. 7(a)-(b), we can also notice that the response of the total intensity is independent of the value of the spin-flip rate. It is interesting to notice the similarity between the response of the dominant polarization in Fig. 7 and early work by Agrawal [22] on the RIN of the main mode and of the side mode of a two-mode semiconductor laser ([22, Fig. 1(a) and (b)]). This similarity can be understood in terms of the study of Erneaux and co-workers [23], that showed that in the limit of large birefringence, the main influence of $\gamma_{s}$ is an increase of cross-saturation gain.

Next, we investigate the role of noise-induced polarization mode hopping, occurring in domains of polarization bistability, when the spontaneous emission noise is large enough. Fig. 8 displays the typical time traces of the total and modal intensities, and Fig. 9 displays the modulation response for the same parameters as Fig. 7, but with stronger noise (in these figures the spontaneous emission noise strength is $\beta_{\mathrm{sp}}=10^{-3} \mathrm{~ns}^{-1}$ ). It can be noticed that polarization hopping mainly affects the modulation response at low frequencies. In the bistability region, Fig. 9(b), both modes are equally dominant and their response is the same 


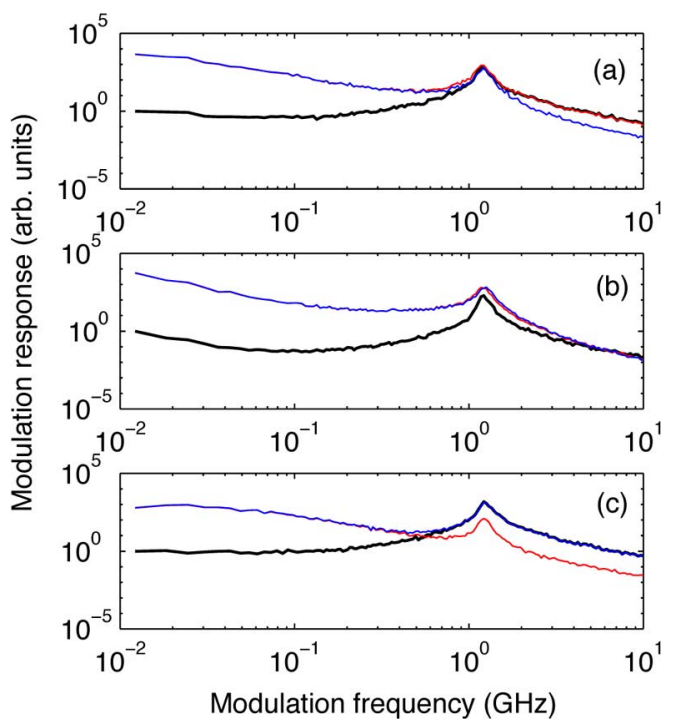

Fig. 9. Modulation response for positive dichroism and large birefringence. The strength of spontaneous emission is $\beta_{\mathrm{sp}}=10^{-3} \mathrm{~ns}^{-1}$, all other parameters are as in Fig. 7.

at low and high frequencies. In contrast, in the monostable regions, the response of both polarizations is the about same at low frequencies, but for frequencies above the relaxation oscillation frequency, the response of the dominant polarization $[x$ in Fig. 9(a), $y$ in Fig. 9(c)] is higher.

\section{DISCUSSION AND CONCLUSIONS}

The modulation response of a free-running VCSEL was numerically investigated using the spin-flip model. We found that in regions of polarization dynamical instability the modulation response exhibits multiple resonance peaks, whose frequencies vary with the injection current and the spin-flip rate. These resonances occur for low values of the spin-flip rate and arise from anticorrelated self-sustained oscillations of the two linear polarizations. We also demonstrated that within regions of stable single polarization the modulation response is as that of a singlemode laser, and is rather unaffected by the value of the spin-flip rate. Since $\gamma_{s}$ affects the coupling of the two polarizations, can be expected that varying $\gamma_{s}$ while keeping the other parameters constant will have an impact on the small-signal modulation response, only in parameter regions where there is polarization competition, and will have no influence on the response, in regions where the laser emits a stable, single polarization. However, if the amplitude of the current modulation is large enough, the response of the VCSEL will depend on $\gamma_{s}$, regardless of the stability of the polarizations in the absence of modulation. Here we focused only on the influence of small amplitude modulation, that can be characterized by the usual small-signal modulation response, that we calculated numerically integrating the full model equations, without linearization. We also investigated the influence of noise-induced polarization mode hopping, that occurs in domains of bistability, when the spontaneous emission noise is large enough. We found that polarization hopping mainly results in a distortion of the modulation response at low frequencies.
It will be interesting to consider, as a natural continuation of the present study, the impact of thermal effects. While long-wavelength VCSELs have several advantages that make them very attractive for data links and optical fiber technologies, they have a relatively large room-temperature gain-cavity spectral misalignment that results in a significant temperature sensitivity. The interplay of thermal effects, polarization switching, and current modulation was studied experimentally and theoretically by Verschaffelt et al. [24]. By modulating the bias current around the PS point and measuring the critical modulation amplitude necessary to force polarization switching, as a function of the modulation frequency, they obtained a so-called polarization modulation frequency response (PMFR) that showed the same cut-off frequency as the thermal frequency response, suggesting the importance of thermal effects. In a follow up study [25] they compared the PMFR of gain-guided and index-guided VCSELs, finding different behaviors: while thermal effects only seemed to play a minor role in the PS of index-guided VCSELs, they seemed to be responsible for the PS in gain-guided devices. These observations were explained within the framework of a rate-equation model [23] that can be derived from the spin-flip model in the limit of large spin-flip rate and large birefringence. The original spin-flip model was extended in [26] to account for the thermal shift of the cavity frequency and the material gain. The investigation of the role of thermal effects in the modulation response, within the framework of this extended spin-flip model, is in progress and will be reported elsewhere.

Our results are of interest for the use of VCSELs emitting on the fundamental transverse mode, in applications where the VCSEL is modulated at a well-defined modulation frequency, since for these applications, a pronounced peak in the modulation response is an attractive feature that results in improved modulation performance.

\section{REFERENCES}

[1] K. Iga, "Surface-emitting laser-Its birth and generation of new optoelectronics field," IEEE J. Sel. Top. Quantum Electron., vol. 6, no. 5, pp. 1201-1215, Sep./Oct. 2000.

[2] H. Thienpont and J. Danckaert, Eds., "VCSELs and optical interconnects," in Proc. SPIE, 2003, vol. 4942.

[3] A. Mircea, A. Caliman, V. Iakovlev, A. Mereuta A, S. Suruceanu, C. A. Berseth, P. Royo, A. Syrbu, and E. Kapon, "Cavity mode-gain peak tradeoff for 1320-nm wafer-fused VCSELs with 3-mW single-mode emission power and 10-Gb/s modulation speed up to 70 degrees C," IEEE Photon. Technol. Lett., vol. 19, no. 1, pp. 121-123, Jan. 2007.

[4] F. Hopfer F, A. Mutig, G. Fiol, M. Kuntz M, V. A. Shchukin, V. A. Haisler, T. Warming, E. Stock, S. S. Mikhrin, I. L. Krestnikov, D. A. Livshits, A. R. Kovsh, C. Bornholdt, A. Lenz, H. Eisele, M. Dahne, N. N. Ledentsov, and D. Bimberg, " $20 \mathrm{~Gb} / \mathrm{s} 85$ degrees C error-free operation of VCSELs based on submonolayer deposition of quantum dots,' IEEE J. Sel. Top. Quantum Electron., vol. 13, no. 5, pp. 1302-1308, Sept./Oct. 2007.

[5] W. Hofmann, M. Gorblich, M. Ortsiefer, G. Bohm, and M. C. Amann, "Long-wavelength (lambda $=1.55 \mathrm{mu}$ m) monolithic VCSEL array with > 3 WCW output power," Electron. Lett., vol. 43, pp. 1025-1026, Sep. 2007.

[6] E. Soderberg, J. S. Gustavsson, P. Modh, A. Larsson, Z. Z. Zhang, J. Berggren, and M. Hammar, "High-temperature dynamics, high-speed modulation, and transmission experiments using 1.3-mu m InGaAs single-mode VCSELs," J. Lightw. Tech., vol. 25, no. 9, pp. 2791-2798, Sep. 2007. 
[7] L. Chrostowski, B. Faraji, W. Hofmann W, M. C. Amann, S. Wieczorek, and W. W. Chow, " $40 \mathrm{GHz}$ bandwidth and $64 \mathrm{GHz}$ resonance frequency in injection-locked $1.55 \mathrm{mu}$ m VCSELs," IEEE J. Sel. Top. Quantum Electron., vol. 13, no. 5, pp. 1200-1208, Sep./Oct. 2007.

[8] X. X. Zhao, D. Parekh, E. K. Lau, H.-K. Sung, M. C. Wu, W. Hofmann W, M. C. Amann, and C. J. Chang-Hasnain, "Novel cascaded injectionlocked 1.55-mu m VCSELs with $66 \mathrm{GHz}$ modulation bandwidth," Opt. Exp., vol. 15, pp. 14810-14816, 2007.

[9] M. San Miguel, Q. Feng, and J. V. Moloney, "Light-polarization dynamics in surface-emitting semiconductor lasers," Phys. Rev. A, vol. 52, pp. 1728-1739, 1995.

[10] J. Martin-Regalado, F. Prati, M. San Miguel, and N. B. Abraham, "Polarization properties of vertical-cavity surface-emitting lasers," IEEE J. Quantum Electron., vol. 33, pp. 765-783, 1997.

[11] M. San-Miguel, , A. Miller, M. Ebrahimzadeh, and D. M. Finlayson, Eds., Semiconductor Quantum Optoelectronics. Bristol, U.K.: Institute of Physics, 1999, p. 339.

[12] Y. Satuby and M. Orenstein, "Small-signal modulation of multitransverse modes vertical-cavity surface-emitting semiconductor lasers," IEEE Photon. Technol. Lett., vol. 10, pp. 757-759, 1998.

[13] Y. Satuby and M. Orenstein, "Mode-coupling effects on the small-signal modulation of multitransverse-mode vertical-cavity semiconductor lasers," IEEE J. Quantum Electron., vol. 35, no. 6, pp. 944-954, Jun. 1999.

[14] M. S. Torre and H. F. Ranea-Sandoval, "Modulation response of multiple transverse modes in vertical-cavity surface-emitting lasers," IEEE J. Quantum Electron., vol. 36, no. 1, pp. 112-117, Jan. 2000.

[15] A. Valle, S. Ortin, and L. Pesquera, "Current modulation of multitransverse mode vertical-cavity surface-emitting lasers," in Pro. 7th ICTON, 2005, vol. 2, pp. 126-131.

[16] C. Masoller, M. S. Torre, and K. A. Shore, "Polarization dynamics of current-modulated vertical-cavity surface-emitting lasers," IEEE J. Quantum Electron., vol. 43, no. 11, pp. 1074-1082, Nov. 2007.

[17] Y. Liu, W. C. Ng, B. Klein, and K. Hess, "Effects of the spatial nonuniformity of optical transverse modes on the modulation response of vertical-cavity surface-emitting lasers," IEEE J. Quantum Electron., vol. 39, no. 1, pp. 99-108, Jan. 2003.

[18] B. Ryvkin, K. Panajotov, A. Georgievski, J. Danckaert, M. Peeters, G. Verschaffelt, H. Thienpont, and I. Veretennicoff, "Effect of photonenergy- dependent loss and gain mechanisms on polarization switching in vertical-cavity surface-emitting lasers," J. Opt. Soc. Amer. B, vol. 16, pp. 2106-2113, 1999.

[19] J. Mulet, C. R. Mirasso, and M. San Miguel, "Polarization resolved intensity noise in vertical-cavity surface-emitting lasers," Phys. Rev. A, vol. 64, 2001, art. no. 023817.

[20] M. Sondermann, M. Weinkath, and T. Ackemann, "Polarization switching to the gain disfavored mode in vertical-cavity surface-emitting lasers," IEEE J. Quantum Electron., vol. 40, no. 2, pp. 97-104, Feb. 2004.

[21] J. Paul, C. Masoller, P. Mandel, Y. Hong, P. S. Spencer, and K. Alan Shore, "Experimental and theoretical study of dynamical hysteresis and scaling laws in the polarisation switching of vertical-cavity surfaceemitting lasers," Phys. Rev. A, vol. 77, 2008, art. no. 043803.
[22] G. P. Agrawal, "Mode partition noise and intensity correlation in a two-mode semiconductor laser," Phys. Rev. A, vol. 37, pp. 2488-2494, 1988.

[23] G. Van der Sande, J. Danckaert, I. Veretennicoff, and T. Erneux, "On rate equations for vertical-cavity surface-emitting lasers," Phys. Rev. A, vol. 67, 2003, art. no. 013809.

[24] G. Verschaffelt, J. Albert, I. Veretennicoff, J. Danckaert, S. Barbay, G. Giacomelli, and F. Marin, "Frequency response of current-driven polarization modulation in vertical-cavity surface-emitting lasers," Appl. Phys. Lett., vol. 80, pp. 2248-2250, 2002.

[25] G. Verschaffelt, J. Albert, B. Nagler, M. Peeters, J. Danckaert, S. Barbay, G. Giacomelli, and F. Marin, "Frequency response of polarization switching in vertical-cavity surface-emitting lasers," IEEE $J$. Quantum Electron., vol. 39, no. 10, pp. 1177-1186, Oct. 2003.

[26] S. Balle, E. Tolkachova, M. San Miguel, J. R. Tredicce, J. Martin-Regalado, and A. Gahl, "Mechanisms of polarization switching in singletransverse-mode vertical-cavity surface-emitting lasers: Thermal shift and nonlinear semiconductor dynamics," Opt. Lett., vol. 24, p. 1121, 1999.

M. S. Torre received the degree of Licenciada en Fisica and the Ph.D. degree from the Universidad Nacional del Centro de la Provincia de Buenos Aires (UNCPBA), Argentina. Her research was primarily in external driven laser physics.

From 1995 to 1997, she was a Postdoctoral Fellow at the Photonics Technology Department, ETSI Telecomunicaciones, Universidad Politécnica de Madrid, Spain. Her research was in quantum-well semiconductor laser modeling. Since 1988 she has been a member of the Quantum Electronic Group of the Physics Institute "Arroyo Seco." She is currently Research Professor at the Facultad de Ciencias Exactas of UNCPBA. Her research interests include modeling and dynamics of VCSEL, dynamics of semiconductor laser with external optical feedback and diffusion effects in semiconductor lasers.

C. Masoller was born in Montevideo, Uruguay, in 1963. She received the M.Sc. degree in physics from the Universidad de la Republica, Montevideo, Uruguay, in 1991 and the Ph.D. degree in physics from Bryn Mawr College, Bryn Mawr, $\mathrm{PA}$, in 1999.

She was Assistant Professor at Universidad de la Republica, Montevideo, Uruguay (1991-2004) working on nonlinear dynamics of semiconductor lasers, multilongitudinal-mode behavior in edge-emitters, polarization competition in VCSELs and optical feedback induced phenomena. Since September 2004 she is a "Ramon and Cajal" researcher at the Departament de Fisica i Enginyeria Nuclear, Universitat Politecnica de Catalunya in Terrassa, Barcelona, Spain. She has co-authored over 65 journal publications. She was co-organizer of three workshops on Chaos, Noise and Complexity in Lasers and Nonlinear Optics, held in Uruguay, in December 2003, 2005 and 2007. Other research interests include theoretical modeling of chaotic systems, synchronization and stochastic phenomena, and nonlinear time-delayed systems. 